\title{
CORTISONE-INDUCED AVASCULAR NECROSIS OF THE FEMORAL HEAD
}

\author{
Richard L. Crless, Montreal, Canada \\ From the Royal Victoria Hospital and McGill University, Montreal
}

\begin{abstract}
Of ninety-five patients with corticosteroid-induced avascular necrosis of bone, ninety-one had involvement of the femoral head. The risk of developing this lesion appeared to be correlated with the total steroid dose. Forty-nine patients demonstrated a subchondral osteolytic defect which healed without proceeding to collapse. Four femoral heads in four patients developed sequestration, resembling osteochondritis dissecans, of a small area of the femoral head. Thirty-eight showed the classical lesion of subchondral osteolysis followed by collapse of the underlying bone. Operation was not necessary in the absence of subchondral collapse. All four patients with the lesion resembling osteochondritis dissecans required surgical replacement of their femoral heads because of disabling symptoms. Twenty of the thirty-eight patients with femoral head collapse required no operation because they either died from their systemic illness or were able to tolerate the level of disability. Eighteen patients required a total of twenty-five operations for disease involving the hips. Varus derotation osteotomy was of benefit in two patients with minimal lesions. When the acetabulum was normal femoral head replacement offered good relief. Total hip replacement has given satisfactory results when the acetabulum showed degenerative changes.
\end{abstract}

Since the first report of steroid-induced avascular necrosis of the head of the femur (Pietrogrande and Mastomarino 1957), the condition has become relatively common. It has caused severe disability in patients who were often systemically ill or who were recovering from renal transplantation. This paper reports a study of the treated by the author. Of these, sixty-eight had had organ transplantation, ten had systemic lupus erythematosus, five had asthma, four had glomerulonephritis, and eight suffered from miscellaneous conditions including Guillan-Barré syndrome, pemphigus, hypopituitarism, sinusitis and convalescence after quadricepsplasty. Of the ninety-five patients with bony lesions, ninety-one had involvement of the femoral

TABLE I

Total Amount of Steroids Administered During Six-month Period After Organ Transplantation

\begin{tabular}{|l|c|}
\hline \multicolumn{1}{|c|}{ Avascular necrosis } & $\begin{array}{c}\text { Total amount of steroids } \\
\text { (gram of prednisone) }\end{array}$ \\
\hline None & $27 \cdot 3 \pm 11 \cdot 2$ \\
Without subchondral collapse & $32 \cdot 6 \pm 9 \cdot 1$ \\
With subchondral collapse & $42 \cdot 3 \pm 14 \cdot 5$ \\
\hline
\end{tabular}

* Significance compared to group with no necrosis: $p<0.01$

natural history of the radiological and pathological lesions of the femoral head and the author's experience in the orthopaedic management of these patients.

\section{MATERIAL}

In the period of eleven years from 1963-74 ninety-five patients with steroid-induced avascular necrosis have been head. Thirty-eight of these had the classical lesion of subchondral osteolysis with subsequent collapse of the underlying bone. Four developed a painful lesion which resembled osteochondritis dissecans. An area of subchondral bone of less than a quarter of the articular surface of the femoral head in either the antero-posterior or the lateral projection became separated from the remainder of the femoral head. In the other forty-nine patients a radiologically 


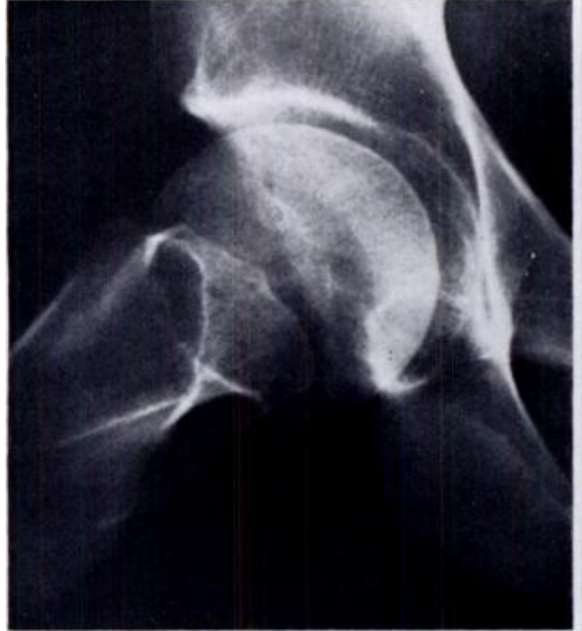

Fig. 1

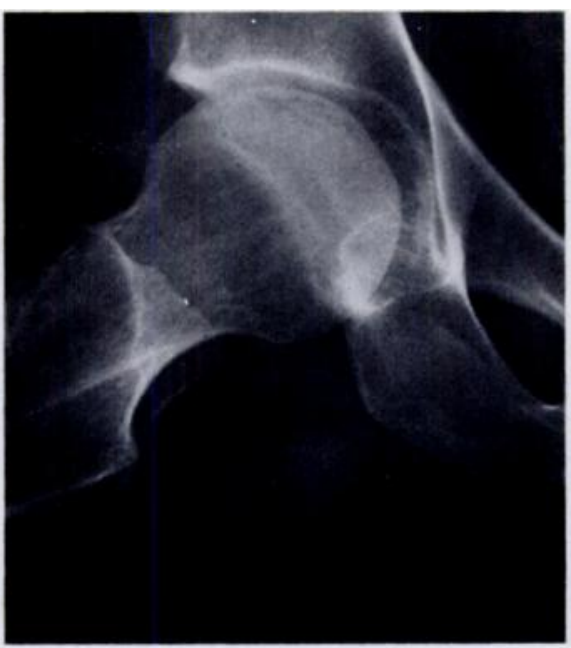

FIG. 2

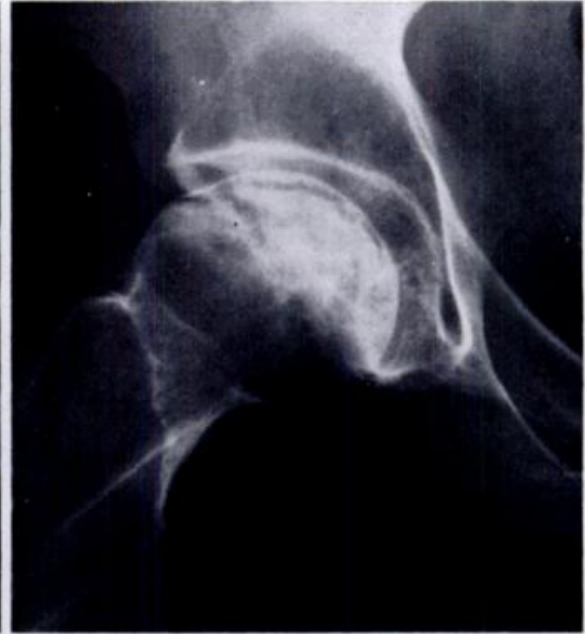

Fig. 3

Lateral radiographs of the hip of a patient after renal homotransplantation. Figure 1-Four and a half months after operation. Small punctate osteolytic areas are seen in the subchondral zone of the femoral head. Figure $2-$ Nine months after transplantation. There has been collapse of the underlying bone with maintenance of the concentricity of the femoral head. As yet there is little sclerosis in the femoral head. Figure 3 Twenty months after transplantation. There has been more extensive collapse and there is sclerosis in the femoral head, probably indicating reparative new bone formation.

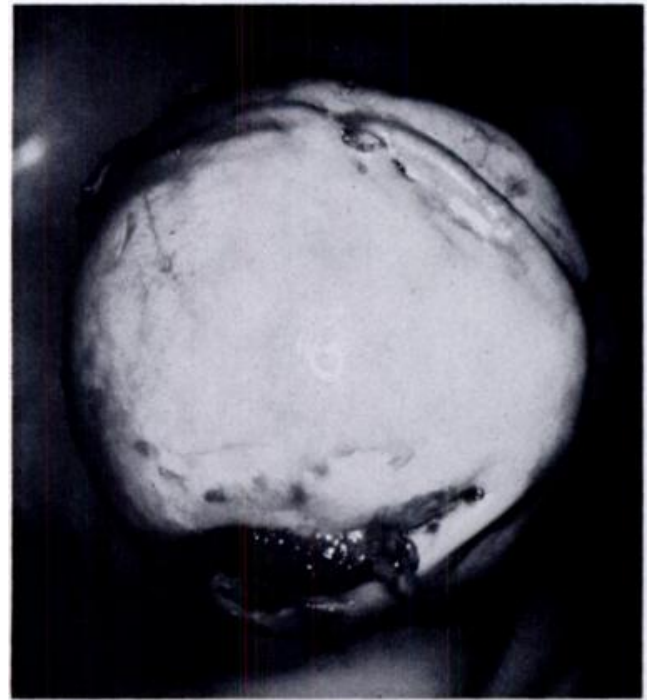

Fig. 4

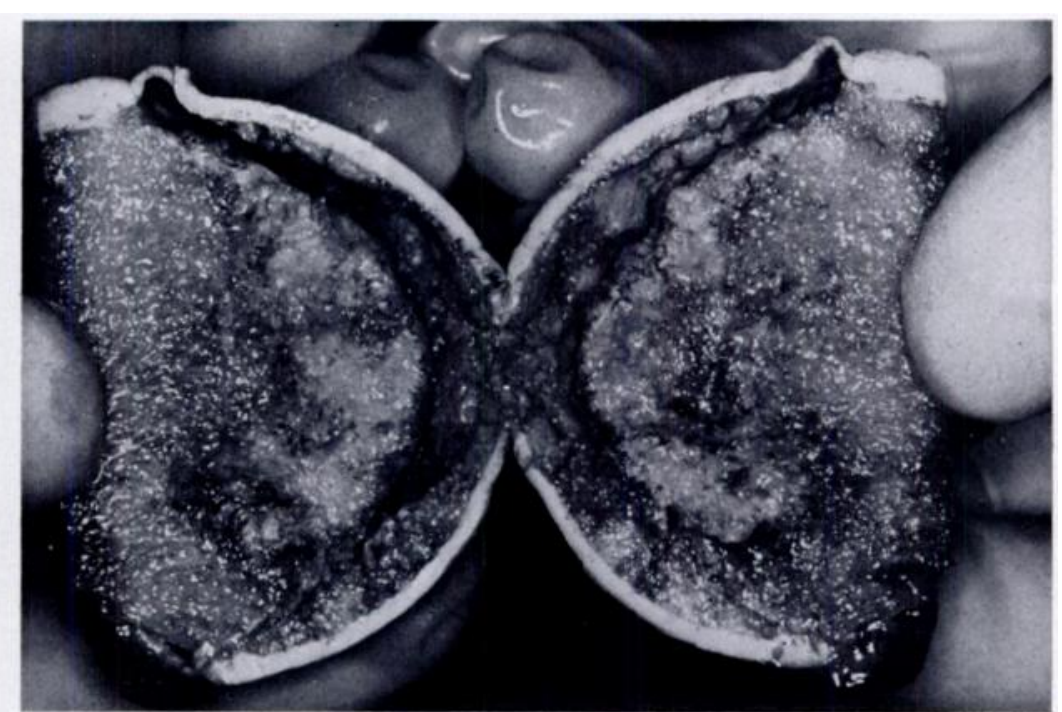

Fig. 5

Figure 4-A femoral head removed eighteen months after renal homotransplantation. A ridge corresponding to the lateral edge of the acetabulum is seen. There is however no break in the articular cartilage. Figure 5-The cut section of the femoral head shown in Figure 4. The intact articular cartilage lies above a space and the collapsed and healing bone is immediately beneath it.

visible lesion showed no collapse and these patients remained free from symptoms. Eighty patients had bilateral lesions. In the forty-two patients who had symptoms (thirty-eight with collapse and four with osteochondritis dissecans) all the lesions were bilateral. With the exception of one patient from the group with symptoms, the time of appearance and progress of the lesions seemed to be approximately equal on both sides. One patient receiving continuous steroid therapy showed radiological and clinical involvement of the second hip fourteen months after the first. The earliest radiological lesion was identified four and a half months after starting cortisone therapy, and the latest appeared at fifteen months. The average time to diagnosis was between nine and ten months.

Other sites of avascular necrosis were found in this group of patients but only four had normal hips in the presence of other bone lesions. The femoral head is the most commonly diagnosed site for steroid-induced avascular necrosis of bone. The humeral head was involved in sixteen patients, the distal femur or proximal tibia in eighteen patients, the talus in six and the capitulum in three. The presence of multiple bone foci obviously complicates the clinical problem.

The relationship of avascular necrosis to the total dose of oral steroids is difficult to determine. In the non-transplant patient, the dosage was poorly recorded and an accurate figure of the total amount of steroids given was unknown. From 1962-1971, a relatively constant immuno-suppressant regime was used for patients after organ transplantation: a baseline drug programme was increased when rejection was threatened. Azothioprine (Imuran) was given in a dose of 4 milligrams per kilogram per day for two weeks, followed by a dose of 3 milligrams per kilogram per day for four weeks. 
Thereafter, it was reduced to 1-2 milligrams per kilogram per day. Prednisone was started at a minimum level of 60 milligrams per day for four to six weeks, and then reduced to 40 milligrams per kilogram for ten days. Thereafter it was reduced by 10 milligram increments every ten days to the lowest tolerated dose, which was never less than $\mathbf{1 0}$ milligrams per day. Actinomycin C (4 micrograms) was given on days lesions received the least amount of steroid during the six-month period. Those showing a lesion without collapse received more and those showing a lesion with collapse received the most. It is concluded that the presence and severity of the lesion is correlated with the total steroid dose, agreeing with the findings of Fisher and Bickel (1971). In this group of patients no correlation was noted between the

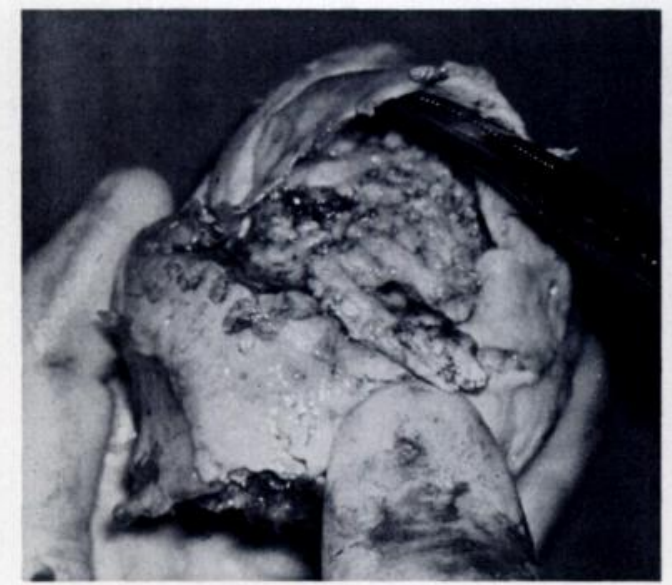

Fic. 6

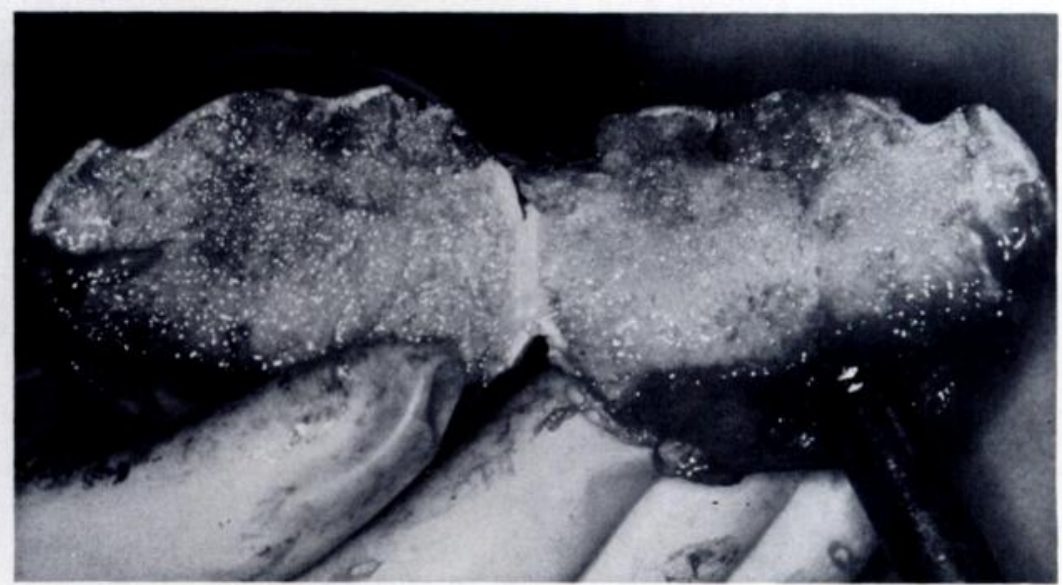

FIG. 7

Figure 6-A femoral head removed fifteen months after beginning steroid therapy for asthma. The separated articular cartilage lies like a cup over the collapsed bone. Figure 7-This femoral head was removed five and a half years after renal homotransplantation. Its earlier changes are shown in Figures 1 to 3 . The articular cartilage has probably become reattached to the underlying bone in a deformed state.

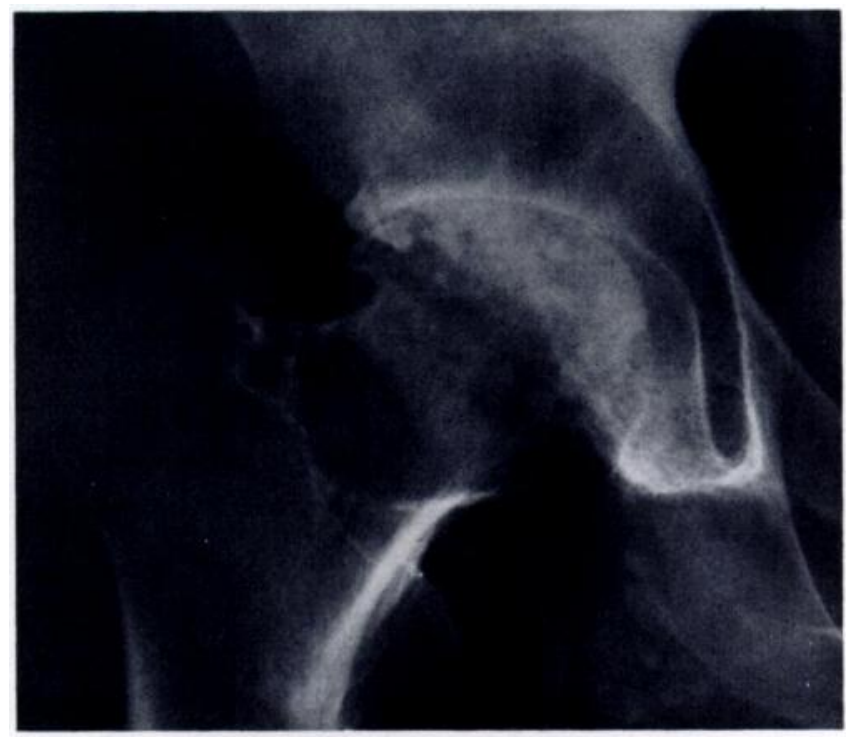

FIG. 8

Eight years after renal homotransplantation a classical degenerative arthritis is shown in a hip which had previously demonstrated the typical natural history of collapse.

one, three, five and seven after operation. During rejection crises prednisone was given in a minimum dosage of 200 milligrams per day for up to two weeks, with additional azothioprine. Several patients had prednisone increased to 500 milligrams per day. Those patients with avascular necrosis were compared with those who showed no such lesion. As can be seen in Table I there was a statistically significant difference between those patients showing avascular necrosis with collapse and those with no radiological evidence of disease. An obvious dose-related trend can be seen; patients with no incidence of the lesion and renal osteodystrophy, but as few patients had been on long-term dialysis, this observation is questionable.

\section{RADIOLOGY AND PATHOLOGY}

\section{Subchondral osteolysis with or without collapse}

As first noted by Merle d'Aubigné, Postel, Mazabraud, Massias, and Gueguen (1965) the first lesion to be seen is a well localised osteolytic area 1 or 2 millimetres in 


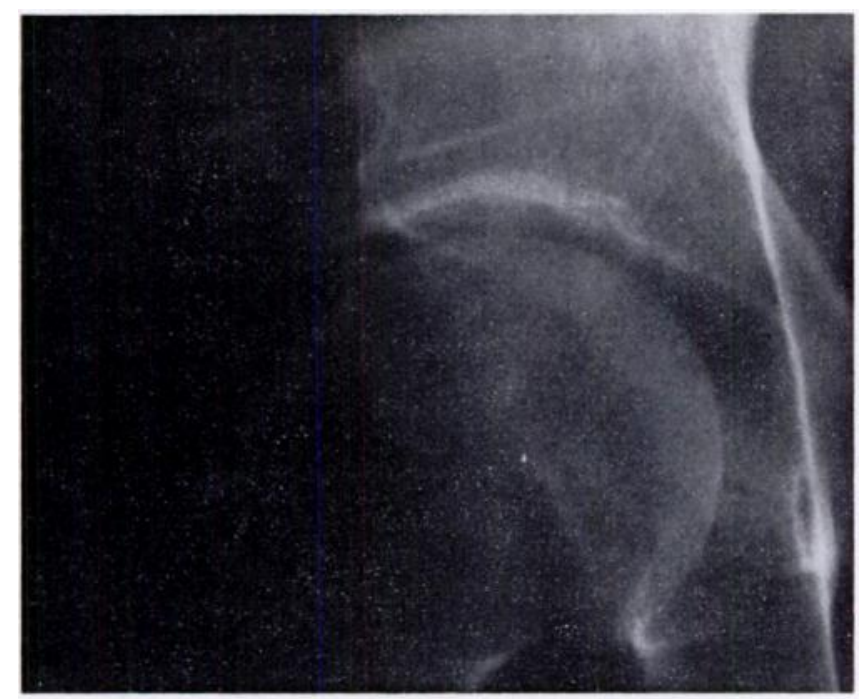

Fig. 9

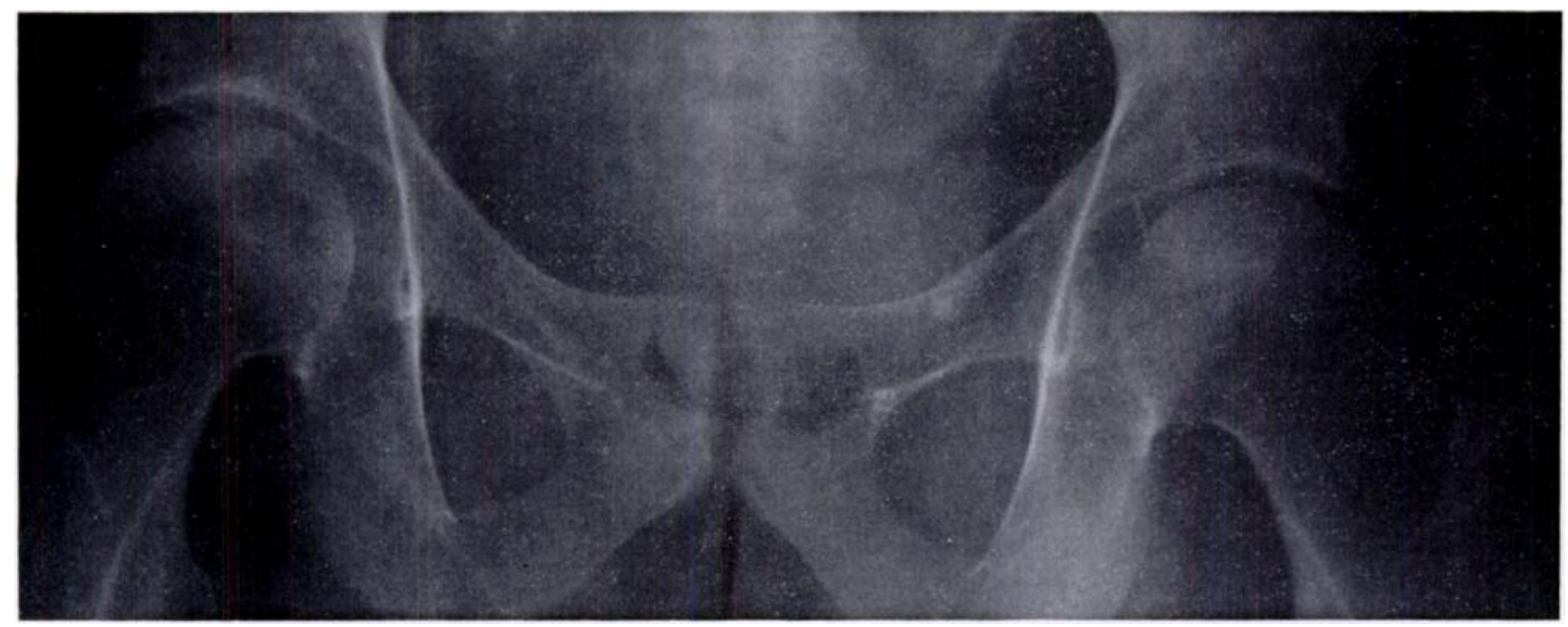

Fig. 10

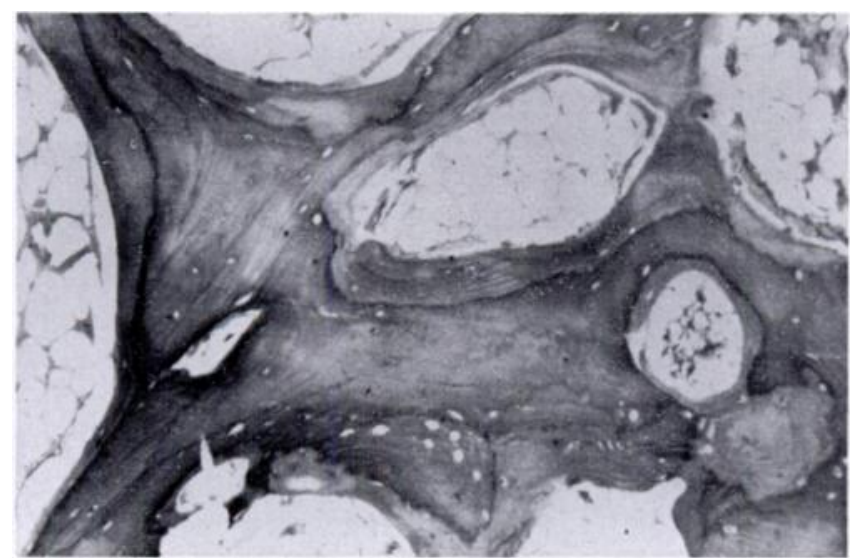

Fig. 11

Radiographic changes in the hip of a patient following renal homotransplantation. Figure 9-Seven months after operation small punctate subchondral osteolytic areas are seen. Figure 10-Thirty-two months after operation a crescentic area of sclerosis is seen in both femoral heads. Figure 11-After forty-eight months the subchondral region of the hip shows necrosis with appositional new bone formation. 
diameter immediately beneath the subchondral bony plate (Fig. 1). There are usually several small osteolytic areas in the subchondral region, and as the condition progresses they tend to coalesce, leaving an area of radiolucency extending beneath subchondral bone for a variable distance. After this, collapse of the underlying bone leads to the appearance of the characteristic "crescent-sign" (Fig. 2). This is explained by Martel, Poznanski and Kuhns (1971) as a vacuum phenomenon whereby nitrogen is drawn into the empty space. As the lesion progresses deformity increases and the collapsed area becomes sclerotic (Fig. 3).

At this time, macroscopic examination shows the articular cartilage to be intact and normal although there is usually a heaped-up ridge of cartilage corresponding to the lateral edge of the acetabulum, the major
Subchondral osteolysis without collapse

Some osteolytic lesions visualised in the subchondral region of the femoral head stay small and do not progress (Fig. 9). The lesion disappears gradually but may leave an area of sclerosis in the femoral head (Fig. 10). Necrotic bone with added appositional new bone has been demonstrated by microscopy in three patients who had such an osteolytic lesion (Fig. 11).

\section{Osteochondritis dissecans}

Subchondral osteolysis has not been seen in a hip affected by osteochondritis dissecans, although in two patients the opposite hip demonstrated subchondral osteolysis with collapse suggesting a similar basic pathology (Fig. 12). An area of subchondral bone

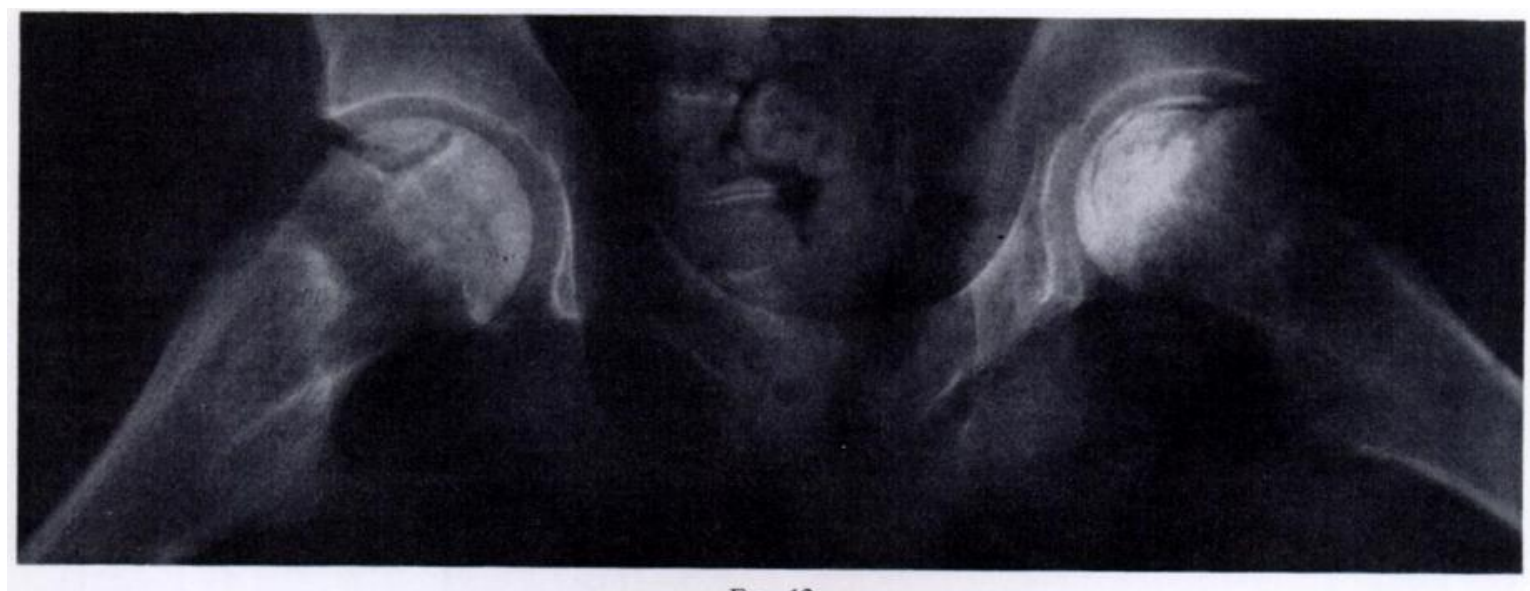

FIG. 12

Eighteen months after renal homotransplantation the right hip demonstrated "osteochondritis dissecans" and the left hip shows classical avascular necrosis with collapse.

deforming force (Fig. 4). Pathologically, the lesion corresponds to that described by Solomon (1973) (Fig. 5). Thus, there is intact articular cartilage with necrotic subchondral bone. Beneath is a space filled with a variable amount of detritus. The collapsed area contains necrotic bone and marrow, and this is bordered by a margin of advancing granulation tissue, which resorbs the necrotic bone and lays down new bone. Beneath this is normal bone and marrow. The acetabulum has not been affected.

The lesion progresses by separation of the articular cartilage from the femoral head. It comes to lie like a cup of cartilage attached only by a small soft-tissue pedicle to the underlying bone (Fig. 6). This fold of cartilage seems to act as a buffer protecting the acetabulum from contact with the deformed bony femoral head.

At a later stage, this loosened articular cartilage becomes reattached to the underlying bone (Fig. 7), but there may then be marked incongruity of the joint, and the cartilage of the femoral head deteriorates. Acetabular damage is then inevitable and degenerative arthritis supervenes (Fig. 8). measuring less than a quarter of the total circumference of the femoral head is separated from the surrounding bone. Microscopy has shown necrotic bone attached to the under surface of intact articular cartilage, and granulation tissue invading the area at the margin of the lesion. The rest of the femoral head appeared normal.

Evidently there is a range of severity of the lesions. Some patients will demonstrate an osteolytic lesion that is not large enough to weaken bone trabeculae and lead to collapse. Others will have a small area of the femoral head separated from the underlying bone but the rest of the femoral head is normal. In others, the subchondral region is more widely involved by the disease process and collapses with separation of the overlying cartilage. This cartilage separates completely, but ultimately becomes reattached to the underlying bone, and degenerative arthritis is the consequence.

Eight femoral heads removed at operation were stained for intravascular fat, according to the method of Jones and Sakovich (1965). As reported elsewhere (Cruess, Ross and Crawshaw 1975) four of these showed intravascular fat in subchondral vessels in regions of necrotic bone. 


\section{CLINICAL FEATURES}

When no collapse occurred, symptoms were absent and the diagnosis was made from radiographs which in most cases were taken for other purposes. Once a hip became painful, the severity of the pain was related to the activities of the patient and to the dose of steroid. Patients who led a largely sedentary life and who received continuous steroid therapy reported a dull ache in the hip at the end of the day and most walked with a limp. The range of hip movement was well preserved.
Subchondral osteolysis with collapse

Thirty-eight patients showed some degree of femoral head collapse. Twenty of these patients required no operation. Eight were seen before extensive collapse had occurred. Reduced activities, crutch walking, and active, isometric joint exercises were advised. Particular emphasis was placed on the prevention of an adduction contracture, as this would increase the likelihood of deformation by the lateral edge of the acetabulum. Of these eight patients, six had had an organ transplant, one

TABLE II

Operations Performed for Cortisone-induced Avascular Necrosis of the Femoral. Head

\begin{tabular}{|l|c|c|c|c|}
\hline & $\begin{array}{c}\text { First } \\
\text { operation }\end{array}$ & $\begin{array}{c}\text { Second } \\
\text { operation }\end{array}$ & $\begin{array}{c}\text { Third } \\
\text { operation }\end{array}$ & Total \\
\hline Varus derotation osteotomy & 2 & - & - & 2 \\
\hline Cup arthroplasty & 2 & - & - & 2 \\
\hline Austin Moore prosthesis & 14 & 2 & - & 16 \\
\hline Total-hip replacement & 2 & 1 & 1 & 4 \\
\hline Resection arthroplasty & - & 1 & - & 1 \\
\hline Total & 20 & 4 & 1 & 25 \\
\hline
\end{tabular}

There was often a palpable or audible click on abduction. The Trendelenburg test was usually positive from an early stage, even when pain was not a prominent feature. If, however, the patient was physically active, even high dose steroid therapy did not prevent disabling symptoms. Femoral head collapse was more extensive than in the more sedentary group of patients. When the steroid dosage was lowered, or discontinued, symptoms became rapidly more severe. This posed a problem in those patients receiving cortisone for reasons other than post-transplantation management. Worst affected were six patients who had received steroid therapy for up to six months after which the dosage had been reduced gradually and discontinued, leaving them with their disabling hip lesions. All these patients required operation to relieve hip pain.

\section{TREATMENT AND RESULTS}

This group of patients presents challenging problems. Individual management is based upon the stage and severity of the radiological lesion, the symptoms, and the life expectancy of the patient.

\section{Subchondral osteolysis without collapse}

The diagnosis of subchondral osteolysis was retrospective, having been made by reviewing radiographs often taken for other reasons. Of the forty-nine patients with this radiological lesion, forty-two were diagnosed in this way. This early lesion requires no treatment. was a severe asthmatic, and one had systemic lupus erythematosus. All cooperated while pain was present, but tended to discontinue this regime as soon as symptoms had subsided. Comparisons of hip radiographs in this group with those of other patients showed no change in the pattern of involvement. Nevertheless relief was often dramatic and consequently this advice is always given to the patient with early symptomatic collapse. It allows time to assess an often changing radiological picture. Of the remaining twelve patients, six died before operation was required. These patients were ill and the musculoskeletal disability was difficult to assess. It is probable that some would have required operation had they survived. Another six patients have continued, some for long periods of time, with hips that have progressed through the collapse to the cartilage reattachment stage, and now have radiological evidence of degenerative arthritis (Fig. 8). All are on continued steroid therapy and none participate in vigorous physical activity. All have some limitation of movement, with medial rotation and abduction particularly restricted. However, flexion is well maintained and the overall functional impairment is small.

Eighteen patients have undergone a total of twentyfive major operations for disease involving their hips. Three patients have had bilateral hip operations. Fifteen patients needed operation on only one hip although all had bilateral hip disease; indeed the radiological picture was not dissimilar in most instances. Patients tended to rely more on the operated hip, thus 


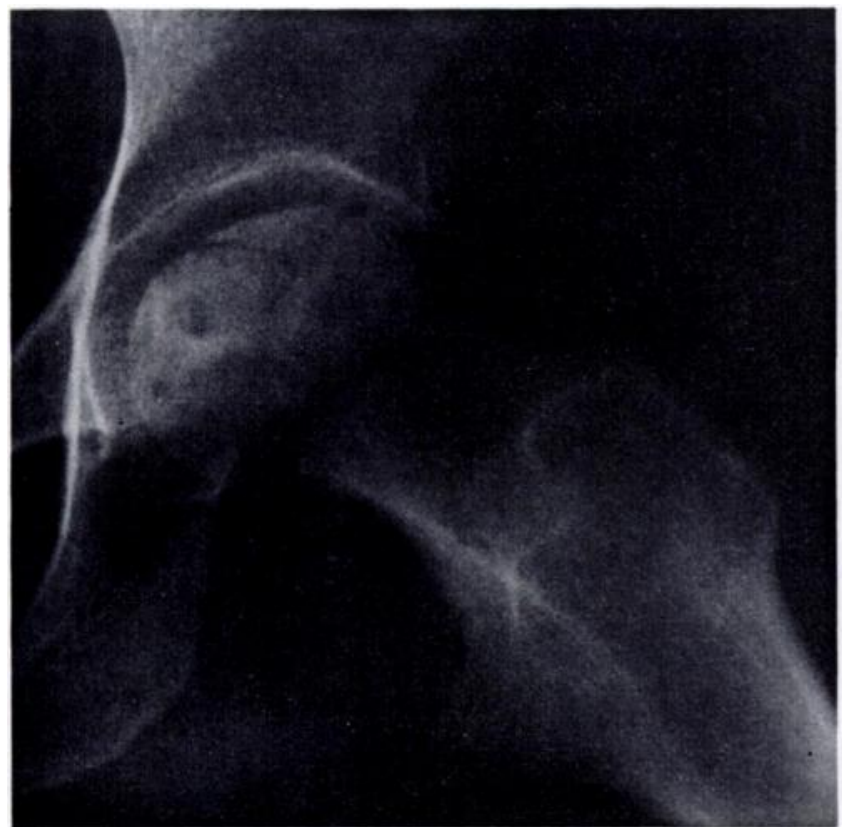

Fig. 13

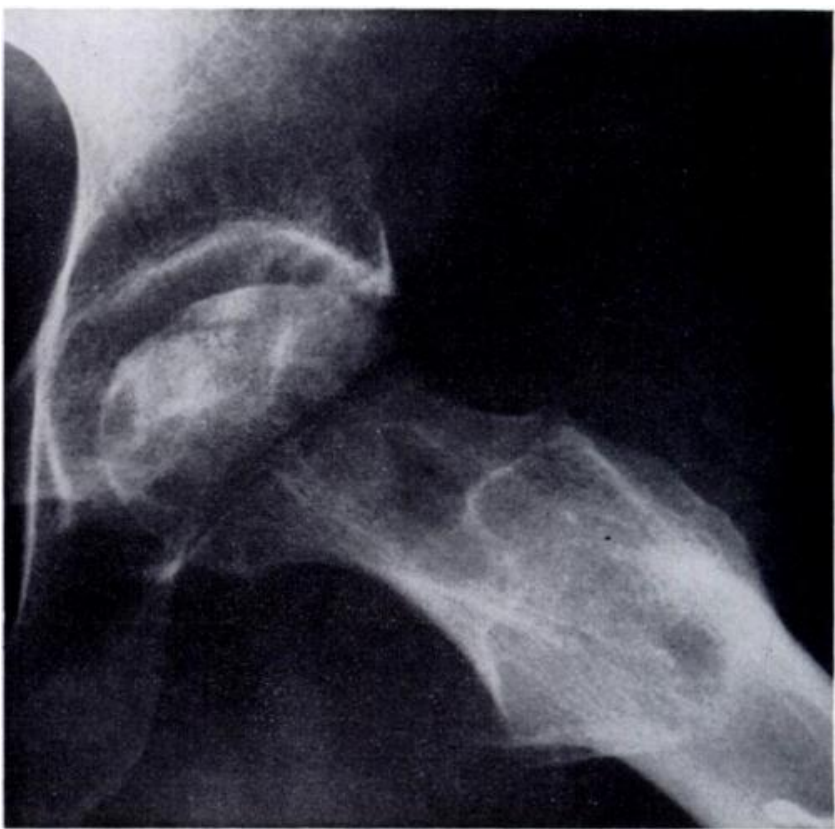

Fig. 14

Figure 13-Eighteen months after commencing steroid therapy for systemic lupus erythematosis, there is evidence of moderate avascular necrosis on the left, with preservation of the outline of the bone. Figure 14-Five and a half years after varus-derotation osteotomy of the hip seen in Figure 13. No further deformity has occurred and some healing is seen. The plate and screws have been removed.

sparing the opposite side and easing symptoms. There were twenty primary operations on hips (Table II). Four failures in the operated group required a total of four secondary operations. One of these secondary procedures also failed, requiring a third operation.

Varus derotation osteotomy-Two patients had this operation, fixation being achieved by a right-angle compression plate (Fig. 13). Their radiographs showed only mild collapse and a varus derotation osteotomy was performed to avoid the lateral edge of the acetabulum deforming the surface of the femoral head. The followup is five and five-and-a-half years. There has been no further collapse in either patient (Fig. 14). Both are free from symptoms and have only 13 millimetres of shortening, easily correctable with a heel raise. Whether this improvement will last remains to be seen but Merle d'Aubigné et al. (1965) report long-term satisfactory results.

Cup arthroplasty-From a theoretical point of view a cup arthroplasty seems an attractive solution in this group of patients, many of whom are young individuals with a life expectancy difficult to determine. Two cup arthroplasties were carried out and both failed at between twelve and eighteen months, with recurrence of pain, progressive limitation of motion and severe restriction of activities. Both patients required further operation, an Austin Moore prosthesis being inserted. Cup arthroplasty is not recommended for this condition, an opinion reflected in other reports (Patterson, Bickel and Dahlin 1964).

Femoral head replacement arthroplasty-Femoral head replacement arthroplasty has been carried out in sixteen patients with a follow-up which now ranges from three to nine-and-a-half years. Fourteen of these were primary operations, and two were carried out for failed cup arthroplasty. This was possible because in neither patient had the normal acetabulum been reamed. The end-result has, in general, been satisfactory. None has

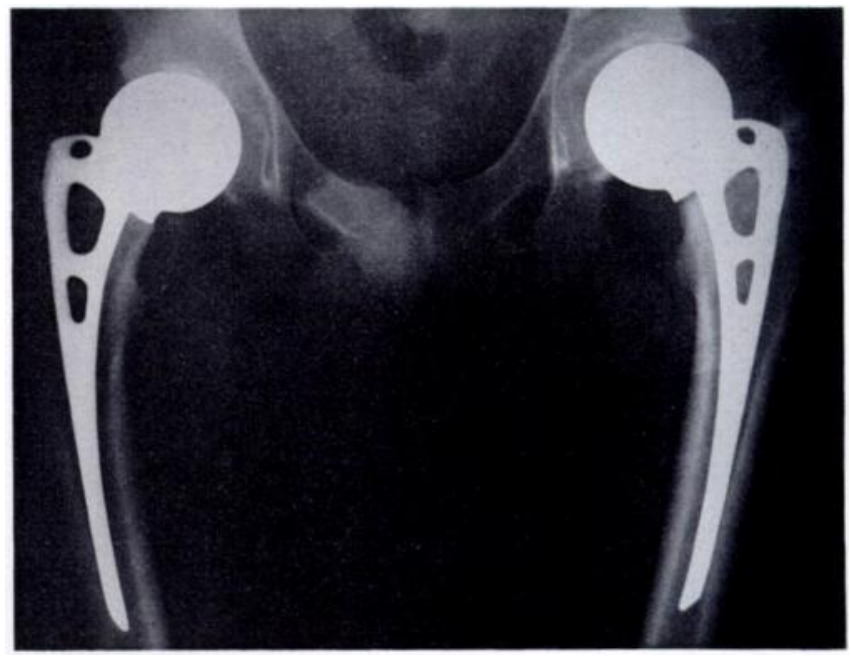

Fig. 15

Fifty-one months after bilateral Austin Moore prostheses have been inserted this patient is working and is free from symptoms.

been classified as excellent, as all have occasional discomfort after periods of intense activity, and all but one have intermittently used a stick. However, twelve of the sixteen are classified as satisfactory with no flexion contracture, a range of flexion to 90 degrees, and abduction greater than 30 degrees. All were able to 


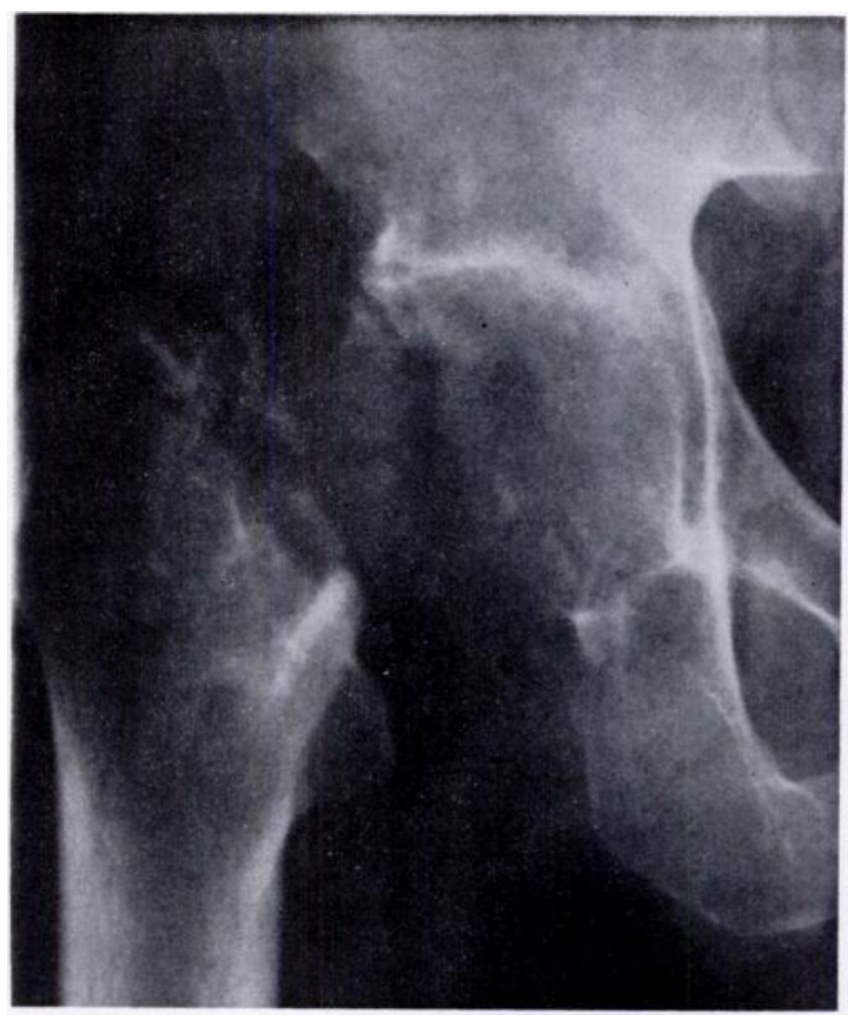

Fig. 16

Extensive soft-tissue calcification or ossification twenty-four months after resection arthroplasty. The hip is stable and the patient is gainfully employed.

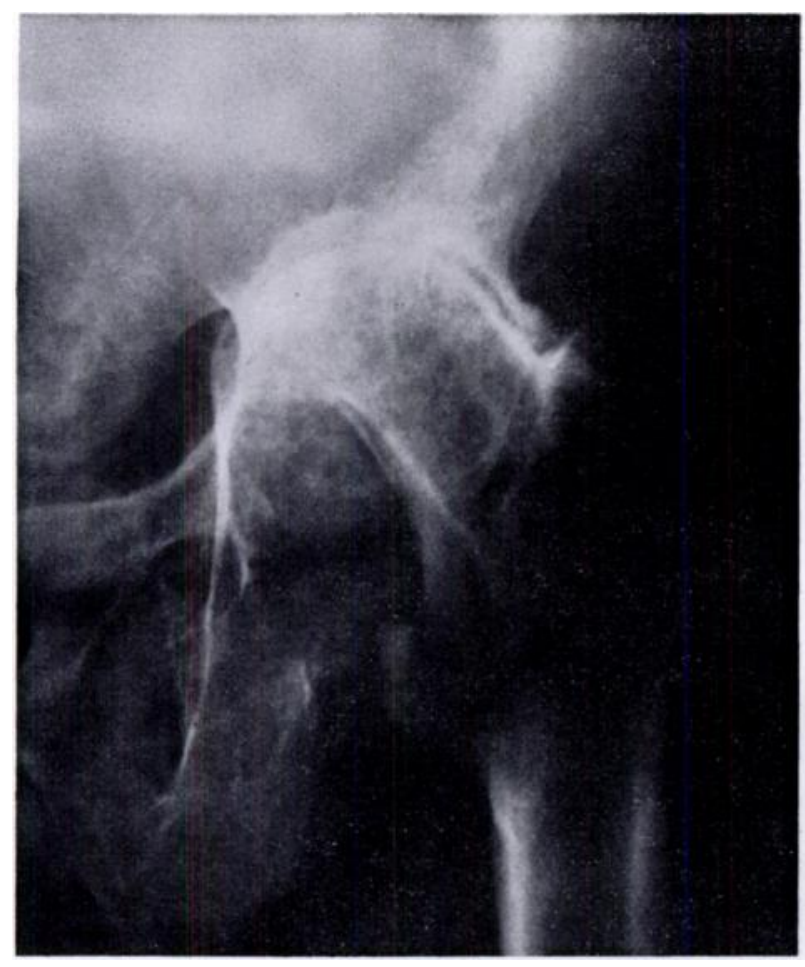

Fig. 17

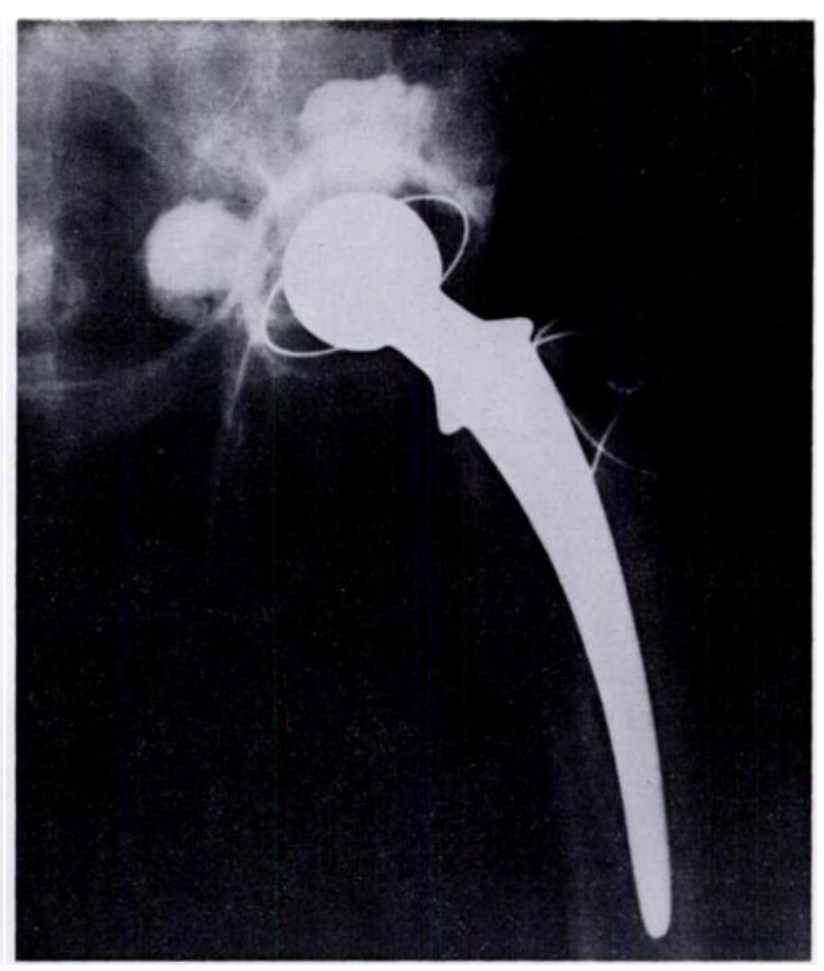

Fig. 18

Figure 17-After high doses of steroid therapy for three years there has been extensive collapse of the femoral head with acetabular damage. Figure 18-Two years after insertion of a Müller prosthesis, the patient is symptom-free and has an excellent end result. 
resume a level of activity consistent with other aspects of their disease (Fig. 15). All showed some loss of acetabular cartilage, but this did not appear to be correlated with symptoms and some joint space was retained in all. The results in these twelve patients did not deteriorate in time. This parallels the experience of Fisher and Bickel (1971).

Four hips in three patients were classified as failures. In the first, there was protrusion of the prosthetic femoral head into the acetabulum resulting in pain and limited activities. The patient died eighteen months after operation with sepsis unrelated to the hip. A second prosthesis protruded into the acetabulum and settled down the femoral shaft. Large amounts of granulation tissue in the acetabulum and within the medullary canal of the femur were found at operation. However, no organism was cultured, probably because the patient was on large doses of systemic antibiotics. The prosthesis was removed and the Girdlestone resection arthroplasty has proved satisfactory. The patient walks with a stick and a shoe raise of 4 centimetres (Fig. 16). The hip flexes to 100 degrees from a flexion contracture of 10 degrees and has active abduction to 40 degrees. He is gainfully employed as a clerk and is free from pain. This represents the only Girdlestone arthroplasty in this series. A third patient has had a total of five operations on her two hips. Her cup arthroplasty failed and was replaced with an Austin Moore prosthesis. Two months later she had an Austin Moore prosthesis inserted on the other side. These two prostheses lasted for eight years. She was twenty-seven years of age at the time of the first operation. During this time she was married, carried on her duties as a housewife and bore one child. She was on intermittent and variable doses of cortisone for asthma throughout this period. Radiologically, there was peri-articular calcification and gradual loss of cartilage. Six and a half years after operation she developed increasing pain and stiffness of both hips. Radiographs demonstrated settling of the prostheses into the pelvis. She consequently has had total replacements on both sides. Total hip replacement - When acetabular damage is inevitable because of extreme deformity of the femoral head, or is established radiologically, total hip replacement is the operation of choice.

Four Müller type total hips have been inserted. Two of these were primary operations, and follow-up is twenty-four and thirty-two months (Figs. 17 and 18). Both patients are classified as excellent in that they have no pain, use no external support, require no analgesics, and have a full range of movement. Both are unilateral procedures.

Two total hip replacements, as mentioned above, have been carried out following bilateral femoral head prosthetic failure. Follow-up is eight and twelve months. One side is classified as excellent, as the patient is pain free with a full range of movement and requires no external support. The second side is the third surgical procedure on the hip and is classified as satisfactory. The patient is pain free, requires no external support or analgesia, but flexion is limited to 80 degrees with no flexion contracture. Abduction is 25 degrees, adduction 20 degrees and total rotation 30 degrees.

\section{DISCUSSION}

Several facts have become clear during the past ten years. In the first place, it is evident that patients receiving systemic corticosteroid therapy are at risk of developing necrosis of bone. This risk is correlated with the total oral steroid dose, and probably with variations in the dosage schedule. The exact amount of cortisone required to cause these lesions is not known. Other related factors are the patients' general health and the disease for which corticosteroids are being given. Fatty embolisation to the subchondral region of bone is probably responsible for the lesion. Steroid administration leads to the development of a fatty liver (Moran 1962). This increase in liver fat is thought to be the source of the hyperlipidaemia which develops. Decreasing the steroid intake may raise the level of lipidaemia (Jones, Engleman and Najarian 1965). The microvascular anatomy of the subchondral zone of the femoral head facilitates embolisation of fat globules (Jones and Sakovich 1966). Cell and marrow death occurs, leading to alteration of function (Fisher, Bickel, Holley and Ellefson 1972; Cruess et al. 1975), and initiating the changes which end in subchondral collapse.

The patients in this series have been treated over an eleven-year period. Many of them, therefore, were managed before the long-term results or exact risks of total hip replacement were known (Charnley and Cupic 1973). From this, as well as other series (Cruess, Blennerhassett, MacDonald, Maclean and Dossetor 1968; Hall, Elmore, Bright, Pierce and Hume 1969; Fisher and Bickel 1971; Harrington, Murray, Kountz and Belzer 1971), it has become apparent that, with care, the steroid-treated patient can undergo major hip surgery with an acceptable risk. At present, it seems that one type of operation is not the answer for all patients suffering from avascular necrosis of the femoral head. Many lesions heal with minimal deformity, and symptoms may not be inconsistent with normal living; consequently, operation should be deferred and a conservative programme outlined, aimed at minimising deformity. In those few patients in whom the radiological lesion is localised to a small area of subchondral bone, and in whom collapse is not extensive, varus derotation osteotomy appears theoretically to maintain the concentricity of the femoral head; it could lead to the arrest of degenerative changes within the hip itself. Whether this arrest will be permanent must await a long-term follow-up of a larger series of 
patients. When collapse is extensive and symptoms warrant surgical intervention, the choice is between femoral head and total hip replacement. Hemiarthroplasty is a shorter operation with less overall risk to the patient. It therefore seems reasonable to recommend hemi-arthroplasty in the young patient with steroid-induced avascular necrosis in whom there is no evidence of acetabular damage. When the acetabular cartilage is damaged the surgeon should opt for total replacement. Successful surgical management of this difficult group of patients requires a knowledge of the disease process, and a proper selection of the correct treatment for each individual patient, depending on his disease and his life-style.

This work was supported by a grant from the Medical Research Council of Canada.

\section{REFERENCES}

Charnley, J., and Cupic, Z. (1973) The nine and ten year results of the low-friction arthroplasty of the hip. Clinical Orthopaedics and Related Research, 95, 9-25.

Cruess, R. L., Blennerhassett, J., MacDonald, F. R., Maclean, L. D., and Dossetor, J. (1968) Aseptic necrosis following renal transplantation. Journal of Bone and Joint Surgery, 50-A, 1577-1590.

Cruess, R. L., Ross, D., and Crawshaw, E. (1975) The etiology of steroid-induced avascular necrosis of bone. A laboratory and clinical study. Clinical Orthopaedics and Related Research, 113, 178-183.

Fisher, D. E., and Bickel, W. H. (1971) Corticosteroid-induced avascular necrosis. Journal of Bone and Joint Surgery, 53-A, 859-873.

Fisher, D. E., Bickel, W. H., Holley, K. E., and Ellefson, R. D. (1972) Corticosteroid-induced aseptic necrosis. II. Experimental study. (linical Orthopaedics and Related Research, 84, 200-206.

Hall, M. C., Elmore, S. M., Bright, R. W., Pierce, J. C., and Hume, D. M. (1969) Skeletal complications in a series of human renal allografts. Journal of the American Medical Association, 208, 1825-1829.

Harrington, K. D., Murray, W. R., Kountz, S. L., and Belzer, F. O. (1971) Avascular necrosis of bone after renal transplantation. Journal of Bone and Joint Surgery, 53-A, 203-215.

Jaffe, W. L., Epstein, M., Heyman, N., and Mankin, H. J. (1972) The effect of cortisone on the femoral and humeral heads in rabbits: an experimental study. Clinical Orthopaedics and Related Research, 82, 221-228.

Jones, J. P., Jun., Engleman, E. P., and Najarian, J. S. (1965) Systemic fat embolism after renal homotransplantation and treatment with corticosteroids. New England Journal of Medicine, 273, 1453-1458.

Jones, J. P., and Sakovich, L. (1965) A technic for staining bone fat. Arthritis and Rheumatism, 8, 448-449.

Jones, J. P., Jun., and Sakovich, L. (1966) Fat embolism of bone. Journal of Bone and Joint Surgery, 48-A, 149-164.

Martel, W., Poznanski, A. K., and Kuhns, L. R. (1971) Further observations on the value of traction during roentgenography of the hip. Investigative Radiology, 6, 1-8.

Merle d'Aubigné, R., Postel, M., Mazabraud, A., Massias, P., and Gueguen, J. (1965) Idiopathic necrosis of the femoral head in adults. Journal of Bone and Joint Surgery, 47-B, 612-633.

Moran, T. J. (1962) Cortisone-induced alterations in lipid metabolism: morphologic and serologic observations in rabbits. Archives of Pathology, 73, 300-312.

Patterson, R. J., Bickel, W. H., and Dahlin, D. C. (1964) Idiopathic avascular necrosis of the head of the femur. Journal of Bone and Joint Surgery, 46-A, 267-282.

Pietrogrande, V., and Mastomarino, R. (1957) Osteopatia da prolungato corticosico. Ortopedia e traumatologia dell'apparato motore, 25, 791-810.

Solomon, L. (1973) Drug-induced arthropathy and necrosis of the femoral head. Journal of Bone and Joint Surgery, 55-B, 246-261. 